$37 \mid 2006$

Langue(s) et religion(s) : une relation complexe dans l'enseignement du français hors de France $x V l^{e}-x X^{e}$ siècle

\title{
Langues et religions. En guise de conclusion : l'enjeu identitaire
}

\section{Willem Frijhoff}

\section{(2) OpenEdition \\ Journals}

Édition électronique

URL : https://journals.openedition.org/dhfles/78

DOI : $10.4000 /$ dhfles.78

ISSN : 2221-4038

Éditeur

Société Internationale pour l'Histoire du Français Langue Étrangère ou Seconde

Édition imprimée

Date de publication : 1 décembre 2006

Pagination : 191-197

ISSN : 0992-7654

Référence électronique

Willem Frijhoff, «Langues et religions. En guise de conclusion : l'enjeu identitaire », Documents pour I'histoire du français langue étrangère ou seconde [En ligne], 37 | 2006, mis en ligne le 02 juillet 2009, consulté le 27 mai 2021. URL : http://journals.openedition.org/dhfles/78 ; DOl : https://doi.org/ $10.4000 /$ dhfles.78 


\title{
Langues et religions \\ En guise de conclusion : l'enjeu identitaire
}

\author{
Willem FRIJHOFF \\ Vrije Universiteit Amsterdam
}

Je voudrais tout d'abord remercier les organisateurs de cette journée, Marie-Christine Kok Escalle et Michel Berré, de l'amitié qu'ils m'ont faite en m'invitant à en être le participant d'honneur. Les communications, riches et diverses, ont suscité beaucoup de questions qui demandent discussion. Je ne voudrais pas frustrer les conférenciers et les auditeurs en anticipant ce débat. Mais il me semble utile de permettre à ces questions de s'insérer dans un cadre interprétatif plus large.

Constatons d'abord que de toute évidence le thème « Langues et religions », examiné en suivant les lieux où les liens entre ces deux dimensions de la vie culturelle - l'école, l'apprentissage, les manuels se nouent, débouche rapidement sur des questions d'identité.

J'esquisserai donc brièvement deux lignes de recherche qui permettront de positionner les communications présentées dans une perspective identitaire. L'identité n'est pas une réalité objective qui existe en dehors de sa perception, ce n'est pas une « chose ». L'on peut définir l'identité comme l'expérience vécue de l'interaction entre l'image de soi et l'imago qu'imposent les autres. La discipline de l'imagologie (la science de la formation, de la gestion et de l'interprétation des stéréotypes, dont aux Pays-Bas le professeur Joep Leerssen est le protagoniste principal) a depuis quelque temps fortement remis en question l'ancienne vision essentialiste de l'identité, qui fait encore fureur dans le domaine politique et dans les médias. 
Historiquement, l'identité s'inscrit en revanche dans un processus continu de formation d'images de soi et de stéréotypes imposés, et sur le plan de la méthode de recherche, dans une perspective de construction symbolique. Autrement dit, l'identité relève d'une double dimension constructiviste, que l'on peut appeler la fabrique communautaire, et l'atelier de l'historien. L'historien cherche dans les sources du passé les formes et instances d'attribution et d'appropriation d'images, tout en sachant que son rôle dans ce processus de recherche n'est pas simplement celui d'un détecteur de formes mais celui d'un architecte qui assure la cohérence des images en lui surimposant cette hyper-image que nous nommons l'identité historique.

\section{La fabrique communautaire}

Pour l'historien, l'identité est une forme de réalisation de soi (le « soi » pouvant être une personne, un groupe, une nation, une culture) qui est inscrite dans des conditions de temps, d'espace et de contexte social. L'identité est par conséquent une valeur qui change continuellement. Elle est fille de la sociolinguistique car les langages de groupe, leurs idiomes, syntaxes et grammaires sociales, jouent un rôle primordial dans la définition de soi, en tant que moi qui se distingue des autres et se différencie dans un contexte donné. En fait, l'identité se construit dans le jeu des groupes identitaires qui se touchent, s'entremêlent et se chevauchent. Chaque personne participe dans une multiplicité de structures publiques et collectives choisies ou imposées qui lui confèrent une identité partielle plus ou moins forte : la famille, le sexe, le groupe d'âge, le village ou la ville, le métier, la religion, la région, la nation, le groupe d'amis, la communauté politique, etc. L'identité d'une personne se compose ainsi d'identités partielles et plurielles qui s'entrecoupent en formant pour chacun une configuration particulière. Dans cette configuration qui reflète très largement les images que les autres se font de nous, l'individu se constitue soi-même une image en privilégiant telle ou telle identité partielle comme dominante dans le domaine public : la nation (l'identité française ou néerlandaise, européenne, cosmopolite, etc.), la religion (catholique, protestant, incroyant, etc.), la langue (francophone, néerlandophone, diglossique, multilinguiste, etc.).

Dans cette formation identitaire, la langue française joue un rôle important pour ceux qui savent parler français, car la langue ouvre non 
seulement un champ de communication possible mais définit tout autant un statut culturel et un niveau de distinction sociale. Ce rôle est bien sûr différent selon que l'on est né en francophonie ou que l'on apprend le français comme langue étrangère ou seconde. Prenons l'exemple du pays où nous nous sommes réunis aujourd'hui, les Pays-Bas. Faisons d'abord remarquer que le français n'y a pas été introduit par les Français de France, mais par des francophones d'autres nations ou régions : au Moyen Age, par exemple, par les Hennuyers du comté de Hainaut, qui depuis 1299 était lié en union personnelle au comté de Hollande mais partageant la même cour princière, puis par les Bourguignons qui avaient repris ces comtés et conquis peu à peu d'autres territoires, mais aussi par les Wallons et Liégeois francophones tout proches. Rappelons que Lille, Arras, Valenciennes, Douai, Dunkerque et Boulogne appartenaient à ce moment encore aux Pays-Bas au sens ancien et large du terme. Les Néerlandais de leur côté fréquentaient non seulement les cours francophones de l'Europe occidentale (Bruxelles, Paris, Dijon, etc.) mais naviguaient sur les côtes françaises (Dieppe, La Rochelle, Bourgneuf, Brouage), commerçaient avec les grandes villes (Paris, Rouen, Lyon, puis Tours, Nantes, Bordeaux) et faisaient leur grand tour vers les villes universitaires françaises (Paris, Orléans, Bourges, Angers, Caen, Poitiers, parfois plus loin). En tant que langue de cour et de commerce le français était supranational.

Dès la fin du XVI ${ }^{\mathrm{e}}$ siècle, la langue française inonda les PaysBas du Nord, en particulier les villes de la Hollande et de la Zélande, à partir des Pays-Bas du Sud. Ce sont les réfugiés wallons du Premier Refuge qui ont eu l'impact le plus fort sur le statut du français en Hollande, et ce sont eux qui en ont fait un emblème identitaire, bien avant les huguenots, bien moins nombreux et beaucoup moins intégrés dans la société d'accueil. L'Eglise wallonne se distinguait d'emblée clairement et consciemment de l'Eglise réformée néerlandaise. Cette distinction, linguistique au début puis peu à peu ecclésiologique et par moments même doctrinale, n'a par la suite cessé de se renforcer et d'englober d'autres dimensions de la vie publique, conférant aux membres de l'Eglise wallonne, néerlandophones d'origine, une distinction sociale qui reflétait un pouvoir politique rehaussé par le prestige de la langue française, devenue entre-temps le véhicule cosmopolite d'une civilisation internationale que les élites au pouvoir avaient eux aussi adoptée. 


\section{Evolutions, fonctions et milieux}

Est-ce un hasard si le sort de la francophonie aux Pays-Bas a suivi un rythme séculaire? De toute façon, à chaque tournant séculaire, la langue française s'est imposée dans une autre fonction et dans un autre secteur de la société néerlandaise, l'ancien Brabant, l'ancienne Flandre et l'ancienne principauté de Liège ayant toujours été des régions bilingues franco-néerlandaises. Examinons rapidement ces tournants privilégiés :

1300 : Union personnelle des comtés de Hainaut, de Hollande et de Zélande (1299), le français entre dans l'aire culturelle du Nord comme une des langues de la cour comtale;

1400 : Les ducs de Bourgogne acquièrent le comté de Flandre (1384) et commencent leur expansion politique mais aussi linguistique dans les Pays-Bas ;

1500 : Essor des relations commerciales entre la France et les Pays-Bas (Anvers); la langue française s'y impose comme langue du commerce international (manuels de commerce, de comptabilité etc.) et conduit à la création d'écoles dites françaises ;

1600 : Le Premier Refuge (depuis 1585), d'ordre confessionnel (quoique pas exclusivement réformé) amène dans les Pays-Bas septentrionaux des dizaines de milliers de francophones des Pays-Bas méridionaux et conduit à l'institution d'Eglises wallonnes et du Synode réformé wallon ;

1700 : Le Second Refuge, huguenot (depuis 1685), consolide l'impact du français dans le monde intellectuel et la République des Lettres, et renforce le statut international du français en Hollande ;

1800: Le français se profile comme la langue des révolutionnaires (1795), des idéaux politiques, puis sous le royaume de Hollande (1806-1810) et l'annexion à l'Empire (1810-1813) comme l'une des deux langues du nouvel Etat centralisé, qui fournit pour longtemps les nouveaux codes législatifs ;

1900: Arrivée en masse des congréganistes catholiques français, rétablissant dans les Pays-Bas les maisons mères et séminaires qui ont été supprimés en France par le pouvoir laïque ; la théologie et la vie catholiques des Pays-Bas seront longtemps marquées de l'empreinte catholique française ;

2000 : L'anglais étant devenu sans conteste la seule lingua franca en Hollande, dans tous les domaines de la vie publique, l'intérêt 
porté à la langue française y est au plus bas, mais des signes avantcoureurs d'un ressaisissement et d'un nouveau rapprochement franconéerlandais se manifestent déjà.

De telles séries rationalisées de chiffres et d'années ne servent bien évidemment qu'à mettre en relief les vicissitudes du français langue étrangère en Hollande et la diversité des situations, fonctions et rôles de la langue, qui entraînent chaque fois un défi identitaire différent. La fonction religieuse du français y était de toutes façons limitée depuis toujours; elle n'est vraiment mise en avant que grâce au retentissement du Second Refuge avec sa multitude d'intellectuels tonitruants. Pensons, par exemple, à la célèbre querelle entre Pierre Bayle et Pierre Jurieu, à Rotterdam. Ces deux figures majeures du monde intellectuel des Lumières radicales (pour emprunter le terme de Radical Enlightenment forgé par les historiens anglophones Margaret Jacob et Jonathan Israel et appliqué par eux à la Hollande de la seconde moitié du XVIII ${ }^{\mathrm{e}}$ siècle) illustrent en tout cas l'importance des intermédiaires, des « interfaces", des passeurs de religions, de savoirs ou de langues entre les sociétés et les cultures, et les bienfaits paradoxaux des émigrations et Refuges.

Toutefois, d'une époque à l'autre et d'une fonction à l'autre, les intermédiaires changent, en fonction des groupes sociaux concernés. Négociants, intellectuels, pasteurs et prêtres, enseignants, courtisans, diplomates et hommes politiques: chaque métier peut fournir ses intermédiaires linguistiques et promouvoir une forme d'identification avec le monde francophone dans son propre secteur identitaire.

\section{L'atelier de l'historien}

L'approche du problème du français langue étrangère ou seconde et de son rapport avec la religion nécessite, en second lieu, une méthodologie proprement historique, tant de la part de l'historien de la langue que du sociolinguiste. Comme il s'agit là de règles du métier qui dépassent de loin la seule portée de ce colloque, je me contenterai ici de quelques remarques à propos des communications présentées.

J'insiste tout d'abord sur l'importance du travail sur les sources. Il ne suffit pas de trouver des textes, encore faut-il les dater finement, les contextualiser à chaque reprise et, lorsque s'agit de textes réédités et réadaptés, suivre leur évolution dans le temps de leur contenu et de leur forme. Quant au contexte, il faut distinguer le contexte social de création 
et de fonctionnement, le contexte d'utilisation, d'apprentissage et d'enseignement, et le contexte social du public visé : la cour, le négoce, la bourgeoisie, les intellectuels, le chant religieux ou profane, les cosmopolites.

D'ailleurs, lorsqu'on analyse les textes, ne faut-il pas se demander plus souvent ce qui est consciemment ou inconsciemment présupposé dans les images que l'auteur veut créer en imposant l'emploi de sa langue et en rédigeant ses textes : l'harmonie sociale ou plutôt la révolution, une morale bourgeoise, l'irénisme confessionnel ou plutôt la lutte contre les hérétiques et autres adversaires, etc. etc. Au cours des siècles, l'évidence de la nation comme cadre d'interprétation croît, au point qu'à l'heure actuelle très peu de Néerlandais échapperaient à l'équation automatique et comme inconsciente entre langue française et nation française. En 1600, par exemple, il en allait probablement tout autrement, le français étant pour le Hollandais de la rue autant langue des Brabançons et des Bourguignons que langue des Français, et au moins autant langue des protestants que des catholiques. Au cours des siècles, ses rapports, qui relèvent étroitement du travail identitaire, se sont peu à peu inversés ou transformés. L'on ne saurait donc se passer d'une interrogation sur le travail de la mémoire dans l'histoire des communautés respectives et de son rapport au travail identitaire.

Enfin, il nous faut nous souvenir de ce que nos textes sont exclusivement des sources verbales. Il faut reconnaître l'évident excès discursif et le surinvestissement linguistique par les auteurs que nous utilisons. Rien d'étonnant en soi, puisque la langue se manifeste dans des textes. Mais la société ne vit bien évidemment pas que de paroles. L'identité se fait et se manifeste autant par la culture visuelle et gestuelle que par la culture verbale, même si depuis toujours la culture néerlandaise se distingue éminemment par son souci de débats et de discussion (comme ma collègue Marijke Spies et moi-même avons essayé de le démonter dans notre synthèse sur la culture néerlandaise du $\mathrm{XVII}^{\mathrm{e}}$ siècle $^{293}$ ). Evitons donc un excès de surinterprétation, en nous efforçant de croiser nos analyses avec celles qui s'appuient sur des sources non verbales.

Je voudrais terminer ces réflexions trop rapides par un appel à un retour à la langue en tant qu'instrument culturel, donc un retour au culturel au sens propre, en mettant l'accent non pas seulement sur les

${ }^{293}$ W. Frijhoff, M.M. Spies, 1650 Bevochten eendracht, Den Haag : SDU, 1999. 
textes, mais sur les pratiques qu'ils mettent en œuvre ou qu'ils supposent, sur les processus sociaux qui les sous-tendent et sur l'action politique qu'ils peuvent provoquer, comme l'a bien démontré la nouvelle histoire des concepts (Begriffsgeschichte). La langue, en effet, n'est pas un élément autonome de la culture, comme le voudrait une vision vitaliste. Elle s'inscrit toujours dans un travail social et culturel sans lequel elle ne saurait être transmise. C'est cette interaction même qui en fait un instrument privilégié pour le travail identitaire. 